\title{
Desempenho coordenativo de crianças: construção de cartas percentílicas baseadas no método LMS de Cole e Green
}

CDD. 20.ed. 152.3

796.07

\section{Resumo}

Os propósitos do estudo foram: 1 ) apresentar valores de referência percentílica às quatro provas da bateria de testes KTK; 2 ) comparar o desempenho coordenativo entre crianças vouzelenses e de outros estudos do pais e exterior; e 3) apresentar pseudo-curvas de velocidade para cada prova. Foram avaliadas 811 crianças com idades entre os seis e os 10 anos. 0 desempenho coordenativo foi estimado por meio da bateria KTK. Cartas percentílicas e pseudo-curvas de velocidade foram construídas com base no método LMS, implementado no "software" LMSchartmarker Pro versão 2.54. Os valores de referência percentílicas expressam forte variabilidade interindividual do desempenho coordenativo. Em geral, os valores médios vouzelenses são inferiores aos belgas e alemães. 0 comportamento do percentil 50 das quatro provas do KTK é similar entre Vouzela, Peru e Açores. As pseudo-curvas de velocidade sugerem especificidade em cada prova e sexo, bem como diminuição dos ganhos coordenativos ao longo da idade.

Palavras-Chave: Coordenação Motora; Cartas de referência; KTK.

\section{Introdução}

A construção de um repertório motor rico e diversificado permite expressar níveis adequados de coordenação motora e assegurar a homogeneidade, integração e unidade estrutural dos diferentes movimentos presentes nas rotinas diárias das crianças, influenciando o seu desenvolvimento psicomotor e aspectos relacionados à sua saúde $\mathrm{e}^{1-5}$.

Níveis adequados de coordenação motora global (grossa) estão condicionados não somente à individualidade biológica, mas também dependem da quantidade e complexidade das experiências motoras adquiridas durante a infância, a que se adicionam aspectos inerentes ao ambiente ${ }^{6-9}$. Nesse processo de desenvolvimento, o contexto escolar torna-se determinante, sendo responsável por favorecer as melhores condições para explorar, ampliar e aprimorar as experiências motoras iniciais, bem como propiciar novas vivências e aprendizados ${ }^{10}$. Não obstante serem fundamentais para o desempenho escolar integral, as experiências estruturadas e supervisionadas de movimento restringem-se, muitas vezes, às aulas de Educação Física, sendo com frequência a única janela de oportunidades de aprendizagem motora orientada.

Em Portugal, proporcionar o desenvolvimento físico e motor adequados à infância é um compromisso consagrado pela Lei de Bases do Sistema Educativo n46/86 e pela Organização da Estrutura Curricular do Ensino Básico. No que concerne aos propósitos da Educação Física, designada por Expressão e Educação Físico-Motora no primeiro ciclo da Educação Básica, elevar o nível funcional das capacidades condicionais e coordenativas é o objetivo-base para o desenvolvimento de todos os blocos de ensino (perícia e manipulação, deslocamentos e equilíbrio, ginástica, jogos, patinagem). E, assim como para todas as áreas de conhecimento, 
deve-se assegurar: 1) a valorização da diversidade de metodologias e estratégias de ensino e aprendizagem; 2) o desenvolvimento de competências em uma perspectiva ao longo da vida; 3 ) e a promoção de ofertas educativas convergentes às diferentes necessidades dos estudantes ${ }^{11}$.

A construção desse contexto de ensino-aprendizagem, significativo ao desenvolvimento da coordenação motora, é um dos grandes desafios da Educação Física. Espera-se do professor não só o desenvolvimento de estratégias e atitudes com exploração ampla das potencialidades motoras, mas também, um conhecimento esclarecido dos diferentes perfis configuracionais que ilustram a variabilidade interindividual. Esses aspectos exigem, necessariamente, avaliação do nível de coordenação motora, identificando trajetórias modais e a variabilidade entre indivíduos no seu desenvolvimento em cada valor discreto de idade, nos anos iniciais da Educação Básica.

Não obstante a diversidade instrumental para avaliar a coordenação motora global, o espaço lusófono tem sido relativamente fértil no uso da bateria de Testes KTK - Köperkoordinationtest für Kin$\operatorname{der}^{12,13}$, sendo significativo no âmbito escolar ${ }^{14-16}$.

Estudos prévios têm utilizado valores percentílicos do desempenho da coordenação motora global baseada no uso da bateria $\mathrm{KTK}^{17,18}$ e coordenaçãao neuromotora baseada no Zurich Neuromotor Assessment ${ }^{19}$ para ilustrar trajetórias modais, bem como a enorme variabilidade interindividual, em cada valor discreto de idade, bem expressa na amplitude de resultados, ou seja, entre os percentis 3 e 97 . Essas pesquisas sugerem valores de referência para populações específicas estudadas, respectivamente, Região Autônoma dos Açores em Portugal (nove ilhas distintas), Lima no Peru (quatro bairros) e Zurique na Suíça.

Com exceção do arquipélago dos Açores, não existe, infelizmente, outra referência portuguesa acerca dos valores percentílicos do desempenho coordenativo de crianças do $1^{\circ}$ ciclo do ensino básico (1. $\left.{ }^{\circ} \mathrm{CEB}\right)$, i.e., anos iniciais do Ensino Fundamental. O Concelho de Vouzela, situado na região centro de Portugal continental apresenta características particulares do território português, com uma interioridade consequente da predominância de áreas rurais com forte variabilidade orográfica e ambiental que a distingue dos grandes agregados habitacionais modernos e industrializados, fortemente urbanizados. A construção de cartas percentílicas locais (i.e., específicas da região) representa um auxílio enorme à gestão sistemática e mais eficiente do processo de ensino e aprendizagem, no âmbito escolar e desportivo, bem como no planejamento de estratégias e intervençōes pelos órgãos institucionais, neste caso, pelas Prefeituras Municipais. É evidente que estes propósitos têm uma elevada generalização a qualquer região, indiferentemente do seu país de origem.

Embora a utilização de cartas percentílicas seja um valioso instrumento de referência, sobretudo no domínio educacional, é possível enriquecê-las ainda mais com a identificação de diferentes janelas de oportunidades para o desenvolvimento da coordenação motora, mediante a identificação, e consequente representação gráfica, dos momentos em que ocorrem os maiores e menores incrementos do desempenho coordenativo, tendo por base a bateria de testes KTK. A apresentação de curvas de velocidade do desempenho coordenativo, designadas por pseudo-curvas de velocidade quando o delineamento da pesquisa é transversal ${ }^{20}$, pode ser especialmente de grande utilidade. Estudos prévios têm reportado a aplicação satisfatória do modelo I de Preece-Baines ${ }^{20,21}$ no estabelecimento de comparações interpopulacionais, a partir de dados transversais, inferindo sobre aspectos do crescimento que, fundamentalmente, exigem um delineamento longitudinal para estimar; por exemplo, a idade no "take-off", a idade do pico de velocidade de altura e a duração do salto pubertário.

Os propósitos da presente pesquisa são os seguintes: 1) apresentar valores de referência percentílica para as quatro provas da bateria de testes KTK; 2) comparar o desempenho coordenativo das crianças vouzelenses com o desempenho das crianças de outros estudos desenvolvidos em locais com estrutura socioeconômica e geográfica bem distinta de Vouzela, como Lima, no Peru ${ }^{18}$, arquipélago dos Açores em Portugal ${ }^{17}$, zona Flamenga da parte norte da Bélgica ${ }^{22}$ e das crianças da pesquisa original desenvolvida na Alemanha nos anos $70^{12}$; 3) apresentar pseudo-curvas de velocidade para cada prova da bateria de testes KTK, representando os momentos de maiores e menores ganhos ao longo dos anos iniciais da Educação Básica. 


\section{Método}

\section{Amostra}

Os participantes desta pesquisa são provenientes do "Projeto Vouzela Ativa", um estudo auxológico e epidemiológico, com delineamento transversal, sobre crescimento somático, desenvolvimento e saúde da população escolar do Concelho de Vouzela, Distrito de Viseu, zona centro de Portugal. Duas amostras foram obtidas em dois momentos distintos, envolvendo todas as 18 escolas municipais distribuídas pelo Concelho. A primeira amostragem refere-se à fase inicial do projeto realizada nos anos 2007 e 2008, na qual foram avaliadas 414 crianças, aproximadamente $93 \%$ da população escolar, com idades compreendidas entre os sete e os 10 anos de idade, pertencentes ao $1^{\circ} \mathrm{CEB}$. A segunda fase do projeto, caracterizada pela continuidade da pesquisa e ampliação do caudal informativo, foi efetuada em 2010 com a avaliação de 397 crianças dos seis aos 10 anos de idade do $1^{\circ} \mathrm{CEB}$, cerca de $90 \%$ da população escolar. A distribuição amostral total está descrita na TABELA 1, em função do sexo e da idade.

O projeto e os protocolos de avaliação foram aprovados pelo Conselho Científico da Faculdade de Desporto, da Universidade do Porto, Portugal. Somente após a assinatura do consentimento livre e esclarecido pelos pais e/ou encarregados de educação dos escolares, uma equipe previamente treinada efetuou as avaliaçóes, a partir de uma abordagem padronizada.

TABELA 1 - Distribuição amostral em função do sexo e da idade

\begin{tabular}{cccccc}
\hline \multirow{2}{*}{ Idades } & \multicolumn{2}{c}{ 1. $^{\text {a }}$ Amostra } & \multicolumn{3}{c}{ 1. $^{\text {a }}$ Amostra } \\
\cline { 2 - 6 } & Meninos & Meninas & Meninos & Meninas & Total \\
\hline 6 & - & - & 39 & 25 & $\mathbf{6 4}$ \\
7 & 41 & 44 & 49 & 45 & $\mathbf{1 7 9}$ \\
8 & 45 & 51 & 47 & 38 & $\mathbf{1 8 1}$ \\
9 & 45 & 51 & 48 & 49 & $\mathbf{1 9 3}$ \\
10 & 72 & 65 & 27 & 32 & $\mathbf{1 9 6}$ \\
Total & $\mathbf{2 0 3}$ & $\mathbf{2 1 1}$ & $\mathbf{2 0 8}$ & $\mathbf{1 8 9}$ & $\mathbf{8 1 1}$ \\
\hline
\end{tabular}

\section{Controle de qualidade da informação}

O controle da qualidade da informação passou por diferentes etapas: 1) treinamento da equipe de avaliação por avaliadores experientes (primeiro e último autores do texto); 2) realização de reteste com uma amostra aleatória de 88 crianças após duas semanas; 3) aplicação das provas sob a supervisão da primeira autora; 4) controle da entrada da informação e análise exploratória prévia para identificar valores extremos; 5) cálculo de estimativas de confiabilidade com base nos resultados pontuais e intervalos de confiança a 95\% do coeficiente de correlação intraclasse (R). Os valores obtidos foram os seguintes: equilíbrio à retaguarda, $\mathrm{R}$ $=0,912(\mathrm{IC} 95 \%=0,772 ; 0,966)$; saltos monopedais, $\mathrm{R}=0,870$ (IC95\% $=0,741 ; 0,935$ ); saltos laterais, $\mathrm{R}$ $=0,873(\mathrm{IC} 95 \%=0,803 ; 0,918)$; transposição lateral, $\mathrm{R}=0,840$ (IC95\% $=0,726 ; 0,906$ ).

\section{Avaliação da coordenação motora}

A avaliação da coordenação motora foi realizada com a bateria de testes Köperkoordinationtest für Kinder (KTK), desenvolvida pelos pesquisadores alemães KipHaRd e SCHILLING ${ }^{12}$. A bateria de testes KTK é constituída por quatro testes: equilíbrio à retaguarda (ER); saltos monopedais (SM); saltos laterais (SL); transposição lateral (TL).

1) ER: caminhar para trás (marcha-ré), três vezes, ao longo de cada uma das três plataformas de equilíbrio, com $3 \mathrm{~m}$ de comprimento, $3 \mathrm{~cm}$ de altura e $6,4,5$ e $3 \mathrm{~cm}$ de largura, respectivamente. Para cada tentativa, são admitidos oito passos, totalizando um máximo de 24 passos para cada plataforma e 72 passos para todo o teste.

2) SM: após um curto período de preparação, saltar a um pé por cima de placas de espuma sobrepostas (pilha crescente, em que cada espuma apresenta $50 \mathrm{~cm} \times 20 \mathrm{~cm} \times 5 \mathrm{~cm}$ ). São atribuídos 3, 2, 1 ponto(s) para o sucesso na execução do teste na primeira, segunda e terceira tentativas, respectivamente. Obtêm-se, no máximo, 39 pontos para cada pé.

3) SL: saltar lateralmente, o mais rápido e o maior número de vezes possíveis, com ambos os pés, sobre uma placa de madeira $(100 \mathrm{~cm} \times 60 \mathrm{~cm} \times 2 \mathrm{~cm})$, dividida por uma régua em madeira $(60 \mathrm{~cm} \times 4 \mathrm{~cm}$ x $2 \mathrm{~cm}$ ). Duração da prova: duas tentativas de 15 segundos. O resultado refere-se ao somatório das duas tentativas.

4) TL: deslocar-se sobre duas placas de madeira $(25 \mathrm{~cm} \times 25 \mathrm{~cm} \times 1,5 \mathrm{~cm})$, de modo intercalado, isto é, em cima de uma das plataformas, mover a outra plataforma, que está ao lado, com as duas mãos para o lado contrário, deslocando-se para esta plataforma de modo sucessivo durante 20 segundos. São efetuadas duas tentativas. O resultado refere-se ao somatório das duas tentativas. 


\section{Procedimentos estatísticos}

Inicialmente foi efetuada a análise exploratória dos dados para a verificação de possíveis erros de entrada de informação ou presença de "outliers", bem como a análise descritiva (média, desvio-padrão e amplitude). A construção das cartas percentílicas foi efetuada, separadamente, para cada uma das quatro provas da bateria de testes KTK e para cada sexo. Os valores das curvas percentílicas foram obtidos pelo método $\mathrm{LMS}^{23}$ implementado no "software" LMSchartmarker Pro versão $2.54^{24}$. Para normalizar a distribuição dos valores em cada uma das variáveis, o método LMS pode assumir a transformação "Box-Cox", específica para cada idade, a dados independentes com valores positivos; os valores L, M e S são "Cubic Splines" em cada intervalo etário. Três curvas suavizadas e específicas de cada idade são produzidas, chamadas de L (transformação "BoxCox"), M (mediana) e $S$ (coeficiente de variação) com base na seguinte equação,

$$
C_{100 \alpha}(t)=M(t)\left[1+L(t) S(t) Z_{\alpha}\right]^{1 / L(t)}
$$

em que $Z_{\alpha}$ é o desvio normal equivalente para a amostra total, $\alpha$ e $C_{100 \alpha}(t)$ o percentil correspondente. A complexidade do alinhamento de cada curva é medida pelos graus de liberdade equivalentes para $L(t), M(t)$ e $S(t)$. Foram utilizados testes $\mathrm{Q}^{25,26}$ para ajuizar a adequação do ajustamento, bem como das representaçōes de "Worm plots"26,27. O melhor modelo é usualmente definido e apresentado por uma série de números relativos aos graus de liberdade. Por exemplo, o L2M4S3o significa que foram necessários 2, 4 e 3 graus de liberdade para as curvas $\mathrm{L}, \mathrm{Me} \mathrm{S}$, e que foi usada a idade original. Em ANEXO I apresentam-se, para cada valor de idade, os valores das curvas $\mathrm{L}, \mathrm{M}$ e $\mathrm{S}$ bem como os respectivos erros-padrão.

\section{Comparação com outros estudos}

A comparação dos níveis de desempenho coordenativo das crianças vouzelenses com o desempenho das crianças de outros estudos será efetuada em dois momentos. Inicialmente serão contrastados os valores médios das quatro provas $\mathrm{da}$ bateria de testes KTK do presente estudo com dois estudos internacionais de referência. $\mathrm{O}$ primeiro refere-se aos valores médios da pesquisa original ${ }^{12}$, desenvolvida na Alemanha na década de 70 , com 1228 crianças e adolescentes, dos cinco aos 14 anos de idade; e o segundo realizado na zona Flamenga, parte norte da Bélgica ${ }^{22}$, publicado recentemente, envolvendo 2470 escolares dos seis aos 11 anos de idade. No segundo momento, será efetuada a comparação do percentil 50, apresentado pelas crianças vouzelenses, açorianas e peruanas. Esses dois estudos, açoriano e peruano, produziram curvas de referência para as provas dessa bateria com base no modelo LMS para crianças de ambos os sexos com idade compreendida entre os seis e os 11 anos. O primeiro apresentou uma amostra de 4007 crianças, enquanto o segundo avaliou 4724 crianças, sendo à data, os únicos a reportarem informação percentílica do desempenho coordenativo a partir da bateria de testes KTK. Importa ressaltar que não apresentaremos a comparação dos valores percentílicos (P50) entre as amostras vouzelense, peruana e açoriana para a prova de TL, devido a uma diferença na contagem da pontuação. De acordo com o manual de testes da bateria $\mathrm{KTK}^{12}$, para pontuar essa prova, atribui-se um ponto para o deslocamento da placa de madeira e outro para o deslocamento do corpo sobre a placa. Nos estudos anteriores ${ }^{17,18}$ foi atribuído somente um ponto a toda trajetória, i.e., transposição da placa e deslocamento do corpo. 


\section{Resultados}

Nas TABELAS 2 e 3 apresentam-se os valores percentílicos (P3, P10, P25, P50, P75, P90, P97) de meninos e meninas em cada valor discreto de idade e para cada prova da bateria de testes KTK. As curvas de referência para o desempenho coordenativo são apresentadas na FIGURA 1, ilustrando o comportamento dos percentis das crianças vouzelenses, de ambos os sexos, com idades entre os seis e os 10 anos, em cada uma das quatro provas. Em todas as provas verifica-se um incremento dos níveis de coordenação motora ao longo da idade, no entanto, a magnitude desse aumento está condicionada ao sexo, sendo específico de cada teste.

TABELA 2 - Valores percentílicos das quatro provas da Bateria de Testes KTK de meninas.

\begin{tabular}{cccccccc}
\hline Equilíbrio à retaguarda & \multicolumn{7}{c}{ Percentis } \\
\cline { 2 - 8 } Idade & $\mathbf{3}$ & $\mathbf{1 0}$ & $\mathbf{2 5}$ & $\mathbf{5 0}$ & $\mathbf{7 5}$ & $\mathbf{9 0}$ & $\mathbf{9 7}$ \\
\hline 6 & 8,42 & 14,68 & 22,12 & 30,62 & 40,08 & 50,43 & 61,62 \\
7 & 9,33 & 16,39 & 24,62 & 33,87 & 44,01 & 54,98 & 66,71 \\
8 & 10,34 & 18,72 & 28,04 & 38,11 & 48,81 & 60,05 & 71,77 \\
9 & 11,90 & 21,35 & 31,33 & 41,70 & 52,37 & 63,30 & 74,45 \\
10 & 12,80 & 23,34 & 33,86 & 44,38 & 54,89 & 65,39 & 75,89 \\
\hline
\end{tabular}

Saltos monopedais

\begin{tabular}{cccccccc} 
& \multicolumn{7}{c}{ Percentis } \\
\cline { 2 - 8 } Idade & $\mathbf{3}$ & $\mathbf{1 0}$ & $\mathbf{2 5}$ & $\mathbf{5 0}$ & $\mathbf{7 5}$ & $\mathbf{9 0}$ & $\mathbf{9 7}$ \\
\hline 6 & 2,32 & 5,96 & 10,81 & 16,72 & 23,59 & 31,35 & 39,95 \\
7 & 3,54 & 7,90 & 13,51 & 20,23 & 27,96 & 36,64 & 46,20 \\
8 & 5,65 & 10,90 & 17,40 & 25,04 & 33,71 & 43,37 & 53,94 \\
9 & 7,54 & 13,50 & 20,74 & 29,12 & 38,58 & 49,05 & 60,48 \\
10 & 9,28 & 15,56 & 23,05 & 31,64 & 41,26 & 51,85 & 63,35 \\
\hline
\end{tabular}

\section{Saltos laterais}

\begin{tabular}{cccccccc} 
& \multicolumn{7}{c}{ Percentis } \\
\cline { 2 - 8 } Idade & $\mathbf{3}$ & $\mathbf{1 0}$ & $\mathbf{2 5}$ & $\mathbf{5 0}$ & $\mathbf{7 5}$ & $\mathbf{9 0}$ & $\mathbf{9 7}$ \\
\hline 6 & 12,86 & 16,94 & 21,55 & 26,68 & 32,32 & 38,47 & 45,13 \\
7 & 14,47 & 19,19 & 24,53 & 30,49 & 37,06 & 44,23 & 52,00 \\
8 & 16,62 & 22,25 & 28,66 & 35,83 & 43,75 & 52,42 & 61,83 \\
9 & 18,39 & 24,87 & 32,28 & 40,59 & 49,80 & 59,90 & 70,88 \\
10 & 19,28 & 26,35 & 34,46 & 43,59 & 53,73 & 64,87 & 77,00 \\
\hline
\end{tabular}

Transposiçáo lateral

\begin{tabular}{cccccccc} 
& \multicolumn{7}{c}{ Percentis } \\
\cline { 2 - 8 } Idade & $\mathbf{3}$ & $\mathbf{1 0}$ & $\mathbf{2 5}$ & $\mathbf{5 0}$ & $\mathbf{7 5}$ & $\mathbf{9 0}$ & $\mathbf{9 7}$ \\
\hline 6 & 18,71 & 22,16 & 25,63 & 29,13 & 32,65 & 36,19 & 39,75 \\
7 & 19,27 & 22,90 & 26,56 & 30,25 & 33,95 & 37,68 & 41,43 \\
8 & 20,12 & 24,03 & 27,98 & 31,95 & 35,95 & 39,97 & 44,01 \\
9 & 21,19 & 25,45 & 29,74 & 34,07 & 38,42 & 42,79 & 47,19 \\
10 & 22,03 & 26,60 & 31,20 & 35,85 & 40,52 & 45,22 & 49,94 \\
\hline
\end{tabular}


TABELA 3 - Valores percentílicos das quatro provas da Bateria de Testes KTK de meninos.

\begin{tabular}{cccccccc}
\hline \multicolumn{2}{c}{ Equilíbrio à retaguarda } & \multicolumn{7}{c}{ Percentis } \\
\cline { 2 - 8 } Idade & $\mathbf{3}$ & $\mathbf{1 0}$ & $\mathbf{2 5}$ & $\mathbf{5 0}$ & $\mathbf{7 5}$ & $\mathbf{9 0}$ & $\mathbf{9 7}$ \\
\hline 6 & 2,54 & 11,63 & 21,58 & 32,03 & 42,83 & 53,91 & 65,22 \\
7 & 4,33 & 13,88 & 24,19 & 34,97 & 46,09 & 57,49 & 69,12 \\
8 & 7,16 & 16,90 & 27,26 & 38,03 & 49,11 & 60,45 & 72,01 \\
9 & 10,05 & 19,81 & 30,10 & 40,75 & 51,68 & 62,86 & 74,23 \\
10 & 12,85 & 22,58 & 32,76 & 43,27 & 54,03 & 65,02 & 76,19 \\
\hline
\end{tabular}

\section{Saltos monopedais}

Percentis

\begin{tabular}{cccccccc}
\cline { 2 - 4 } & $\mathbf{3}$ & $\mathbf{1 0}$ & $\mathbf{2 5}$ & $\mathbf{5 0}$ & $\mathbf{7 5}$ & $\mathbf{9 0}$ & $\mathbf{9 7}$ \\
\hline 6 & 1,91 & 7,18 & 13,48 & 20,45 & 27,95 & 35,89 & 44,21 \\
7 & 3,13 & 9,26 & 16,42 & 24,30 & 32,74 & 41,65 & 50,97 \\
8 & 5,20 & 12,27 & 20,33 & 29,11 & 38,47 & 48,32 & 58,59 \\
9 & 6,98 & 14,60 & 23,17 & 32,44 & 42,29 & 52,63 & 63,40 \\
10 & 8,43 & 16,13 & 24,69 & 33,91 & 43,67 & 53,90 & 64,54 \\
\hline
\end{tabular}

Saltos laterais

\begin{tabular}{cccccccc} 
& \multicolumn{7}{c}{ Percentis } \\
\cline { 2 - 8 } Idade & $\mathbf{3}$ & $\mathbf{1 0}$ & $\mathbf{2 5}$ & $\mathbf{5 0}$ & $\mathbf{7 5}$ & $\mathbf{9 0}$ & $\mathbf{9 7}$ \\
\hline 6 & 16,13 & 20,50 & 25,56 & 31,37 & 37,96 & 45,37 & 53,66 \\
7 & 17,28 & 21,97 & 27,39 & 33,61 & 40,67 & 48,62 & 57,49 \\
8 & 18,79 & 23,88 & 29,78 & 36,54 & 44,21 & 52,85 & 62,50 \\
9 & 20,32 & 25,83 & 32,21 & 39,52 & 47,82 & 57,16 & 67,60 \\
10 & 21,56 & 27,40 & 34,17 & 41,93 & 50,74 & 60,65 & 71,72 \\
\hline
\end{tabular}

Transposiçáo lateral

\begin{tabular}{cccccccc} 
& \multicolumn{7}{c}{ Percentis } \\
\cline { 2 - 8 } Idade & $\mathbf{3}$ & $\mathbf{1 0}$ & $\mathbf{2 5}$ & $\mathbf{5 0}$ & $\mathbf{7 5}$ & $\mathbf{9 0}$ & $\mathbf{9 7}$ \\
\hline 6 & 19,27 & 23,39 & 27,59 & 31,86 & 36,21 & 40,62 & 45,08 \\
7 & 20,08 & 24,32 & 28,65 & 33,06 & 37,54 & 42,08 & 46,67 \\
8 & 21,12 & 25,50 & 29,98 & 34,54 & 39,16 & 43,86 & 48,61 \\
9 & 22,14 & 26,66 & 31,28 & 35,98 & 40,75 & 45,59 & 50,49 \\
10 & 23,02 & 27,65 & 32,37 & 37,17 & 42,05 & 47,00 & 52,01 \\
\hline
\end{tabular}



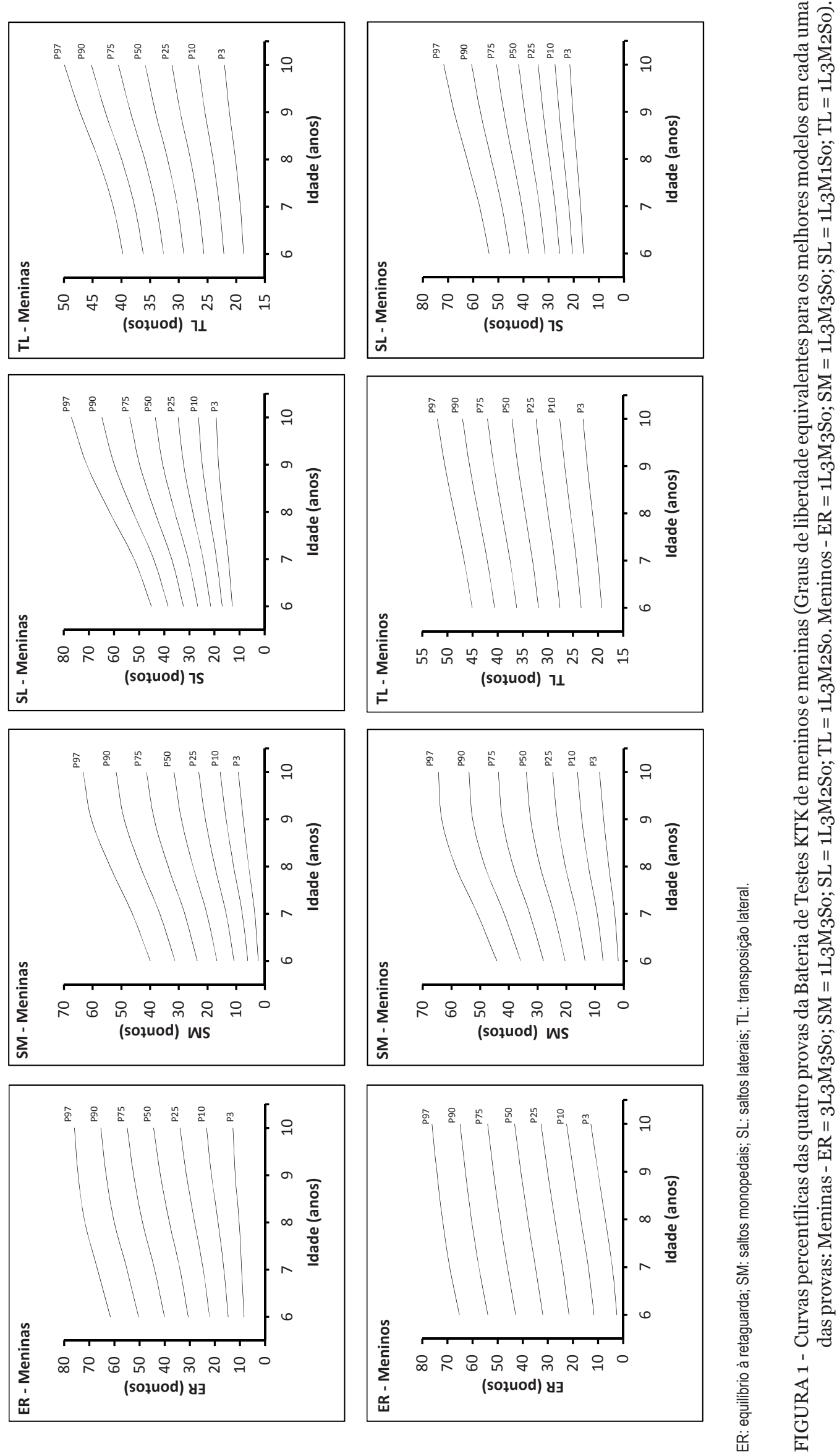

Rev Bras Educ Fís Esporte, (São Paulo) 2013 Jan-Mar;27(1):25-41 • 31 
A FIGURA 2 ilustra o comportamento dos valores médios das quatro provas da bateria de testes KTK das crianças vouzelenses em comparação às médias reportadas em dois estudos de referência internacional, realizados na Bélgica e na Alemanha, referidos anteriormente. Em geral, as crianças vouzelenses evidenciam valores médios inferiores nas provas SM, SL e TL, sobretudo nos SM. Nos testes $\mathrm{SM} \mathrm{e} \mathrm{TL}$, as crianças vouzelenses apresentam menores incrementos ao longo da idade, contrariamente ao observado nas outras duas amostras. Em três provas, nomeadamente SM, SL em ambos os sexos e ER nas meninas, verifica-se um ligeiro declínio entre os nove e 10 anos na amostra vouzelense, enquanto as crianças belgas e alemãs parecem manter os incrementos médios na pontuação dos testes. $\mathrm{Na}$ prova do ER, o comportamento dos valores médios distingue-se dos demais testes. Entre os seis e os sete anos de idade, as médias dos meninos vouzelenses são superiores aos belgas, estando próximos aos valores alemães. A partir dos oito anos, os valores médios dos meninos vouzelenses assemelham-se aos belgas, assim como as médias das meninas entre os seis e os nove anos de idade.

A FIGURA 3 apresenta o contraste do comportamento do P50 em três provas da bateria de testes KTK entre três amostras: vouzelense, peruana e açoriana. Relativamente à prova do ER, as meninas das três amostras apresentam valores semelhantes ao longo da idade. Os meninos vouzelenses mostram menor incremento ao longo da idade, enquanto os açorianos evidenciam maior declive da reta entre os seis aos 10 anos. Nos SM, os maiores valores do P50 são das crianças peruanas, embora, a partir dos oito anos de idade, as meninas açorianas apresentem valores muito próximos, sobrepondo as retas. Nos SL, as crianças vouzelenses mostram menores incrementos ao longo da idade, comparativamente às peruanas e açorianas.

Na FIGURA 4 estão representadas, graficamente, as pseudo-curvas de velocidade da mediana, em função do sexo, referente às quatro provas da bateria de testes KTK. Em todas as pseudo-curvas, identifica-se uma diminuição da magnitude de ganhos anuais, sendo específicas de cada prova, bem como diferentes em meninos e meninas. Nas meninas, as pseudocurvas de velocidade das provas ER, SM e SL são similares e evidenciam maiores taxas de incrementos anuais entre os seis e os sete anos de idade; na TL, a maior velocidade de desempenho coordenativo parece ocorrer entre os oito e os nove anos. Nos meninos, cada prova apresenta um comportamento distinto em função das oscilações aumento/redução das velocidades de desempenho, embora também se verifiquem maiores resultados entre os seis e os sete anos e menores aos 10 anos de idade. 


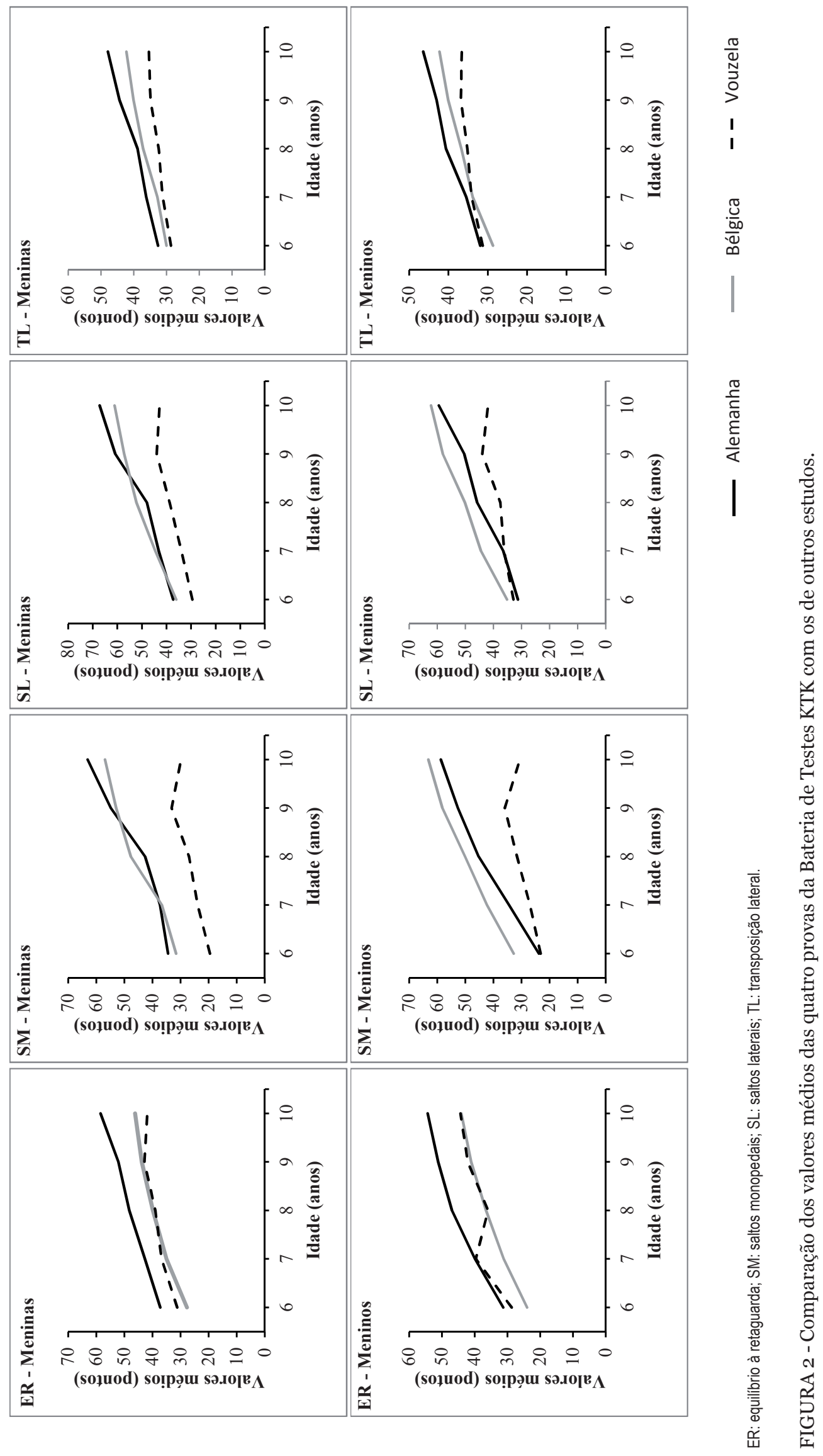

Rev Bras Educ Fís Esporte, (São Paulo) 2013 Jan-Mar;27(1):25-41 • 33 

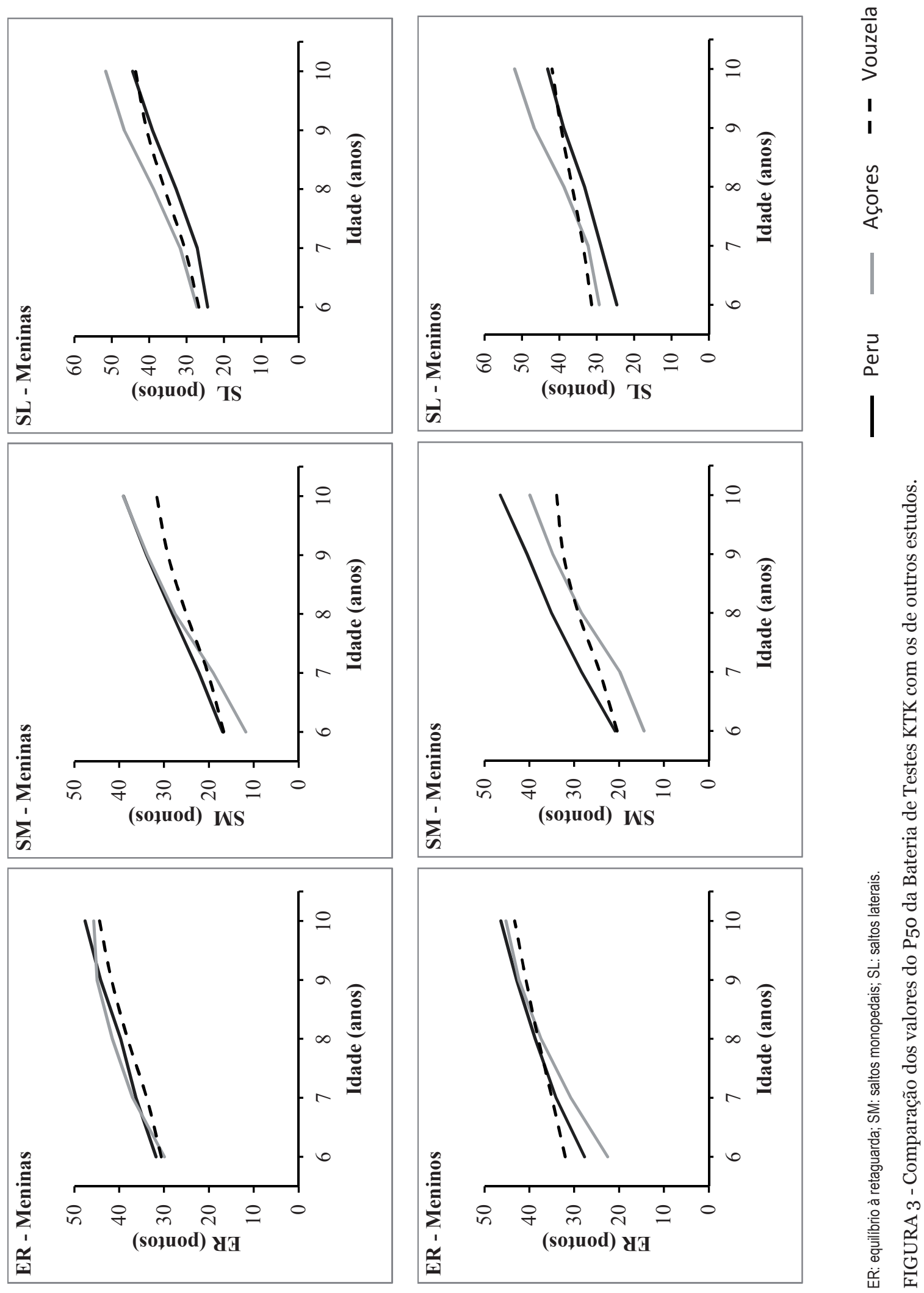

34 • Rev Bras Educ Fís Esporte, (São Paulo) 2013 Jan-Mar;27(1):25-41 

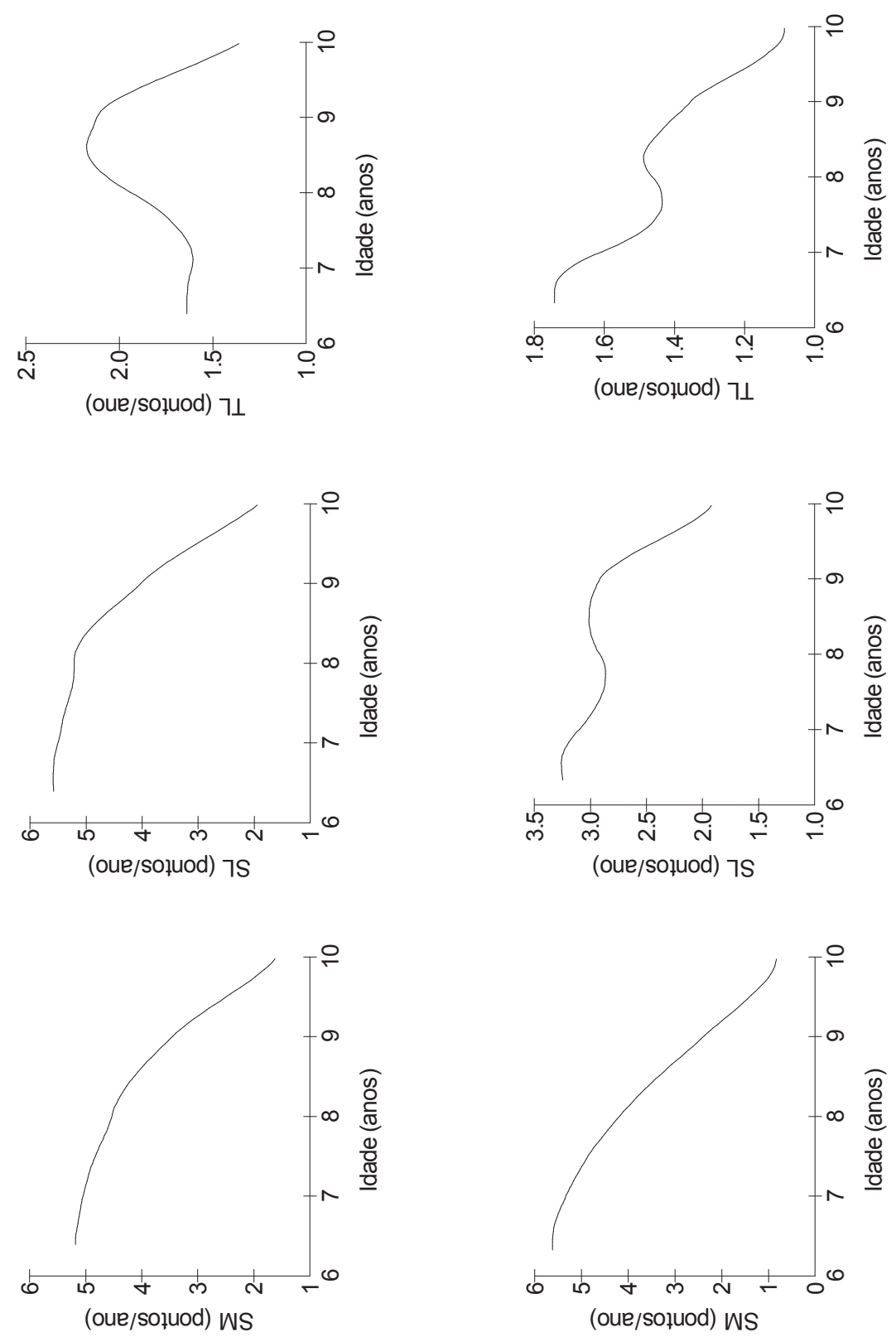

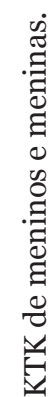
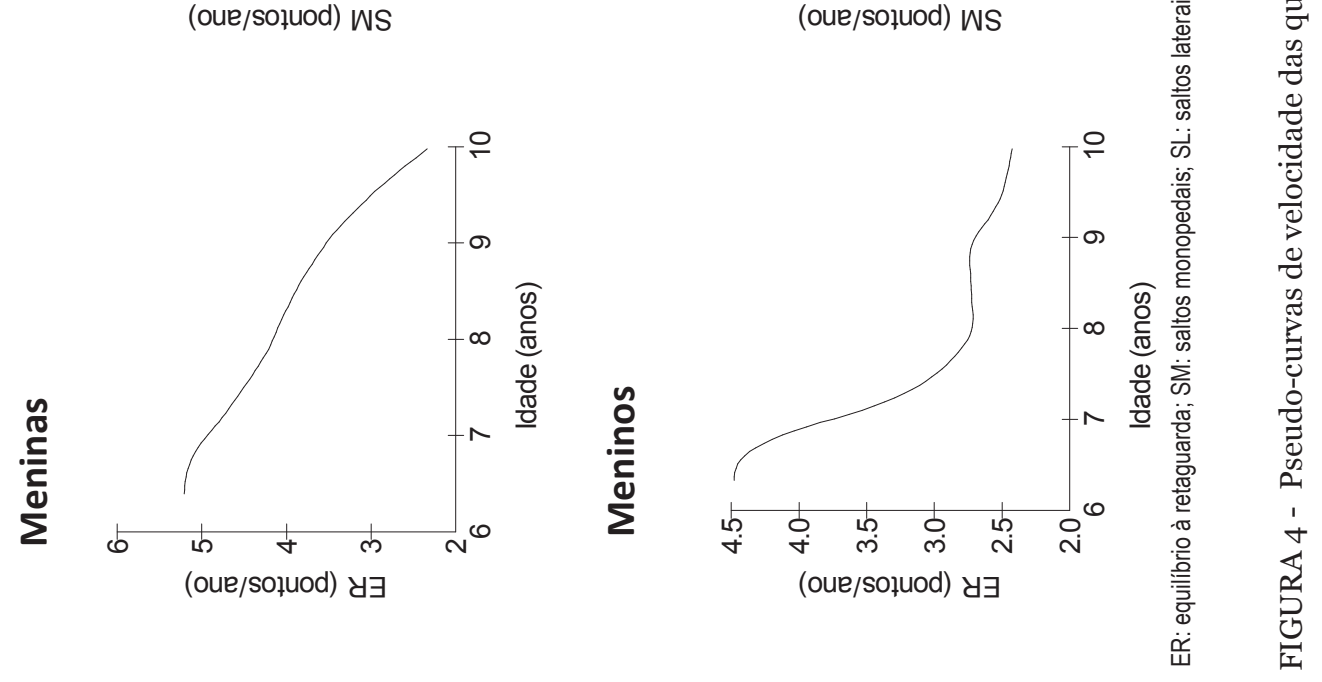

Rev Bras Educ Fís Esporte, (São Paulo) 2013 Jan-Mar;27(1):25-41 • 35 


\section{Discussão}

Este estudo teve como objetivos construir valores de referência percentílica para as provas da bateria de testes KTK, comparar o desempenho coordenativo das crianças vouzelenses com o desempenho das crianças de estudos desenvolvidos em locais com estruturas socioeconômicas e geográficas distintas, a que se adicionou a apresentação de pseudo-curvas de velocidade. São intentos de confluência entre inquietação acadêmica e significado educacional no contexto de intervenção pedagógica em crianças do $10^{\circ} \mathrm{CEB}$.

As cartas centílicas de referência apresentadas têm a sua origem em um delineamento transversal, cujo plano de amostragem assegura a representatividade das crianças do Concelho de Vouzela, com idades compreendidas entre os seis e os 10 anos, proveniente das 18 instituiçôes municipais do $1 .{ }^{\circ} \mathrm{CEB}$, refletindo, por isso, diferentes condiçôes geográficas e sociais do Concelho. Salienta-se, ainda, o considerável número de sujeitos em cada valor discreto de idade, exceto aos seis anos. Há informações provenientes de estudos de simulação para construção de cartas centílicas de altura $^{28}$, em que é sugerido o valor de 300 sujeitos por idade e sexo, sobretudo quando se pretende obter estimativas precisas dos percentis extremos (P3 e P97). Não obstante essa sugestão estrita no domínio da Auxologia, há resultados disponíveis na literatura relativos à elaboração de cartas de referência do desempenho neuromotor com uma dimensão amostral não muito distinta da referida no presente estudo. Assim, LARGO et al. ${ }^{19}$ reportaram valores percentílicos para o desenvolvimento neuromotor com base em uma amostra de 662 crianças e adolescentes suíços com idades compreendidas entre os cinco e os 18 anos, i.e., 47 sujeitos em cada valor discreto de idade, independentemente do sexo. Em outro estudo, já no domínio da composição corporal, desenvolvido no Sudeste da Inglaterra ${ }^{29}$ relativo à elaboração de curvas de referência da massa gorda corporal total, foram amostrados 1985 indivíduos, também dos cinco aos 18 anos de idade, ou seja, aproximadamente 71 sujeitos em cada idade e sexo. Ora, o problema reside, no nosso estudo, na amostra aos seis anos de idade, 39 meninos e 25 meninas, 64 no total, não muito distinta dos estudos anteriores, a que acresce o fato da qualidade das curvas $\mathrm{L}, \mathrm{M}$ e $\mathrm{S}$ nesta idade ser relativamente precisa face aos valores dos erros-padrão serem baixos e praticamente iguais aos das outras idades.

O uso do método LMS para expressar a variabilidade interindividual explícita no desempenho coordenativo tem apresentado objetividade suficiente e vantagens claras relativamente a outros métodos de estimação ${ }^{30}$. Destaca-se a distinção na representação gráfica das curvas de referência e o modo como as constrói, suavizando a distribuição dos valores e mantendo equilíbrio entre a fidedignidade dos dados e a parcimônia dos modelos ajustados. No que se refere à confiabilidade dos valores do desempenho nas quatro provas da bateria de testes KTK, os valores da correlação intraclasse foram elevados, entre 0,84 e 0,91 , corroborando estudos prévios e mostrando a reduzida variância de erro; por exemplo, LOPES et al. ${ }^{14}$, em um estudo com crianças açorianas, reportaram estimativas situadas entre 0,78 e 0,98 , e VALDIVIA et al ${ }^{18}$, em estudo com crianças peruanas, referiram valores entre 0,80 e 0,91 . Relativamente à parcimônia no ajustamento dos modelos para cada prova e sexo, a partir dos três parâmetros escalares relativos aos graus de liberdade (E) de cada curva, i.e., EL, EM, ES, sugeridos por Cole e GREEN ${ }^{23}$, os modelos finais refletem o melhor ajustamento e estão de acordo com a proposta de PAN e ColE ${ }^{24}$.

As curvas percentílicas expressam a forte variabilidade interindividual em cada valor discreto de idade no desempenho das quatro provas da bateria de testes KTK, sendo distinta entre meninos e meninas e entre testes. Por exemplo, na prova dos SL, a amplitude dos valores percentílicos aos seis anos varia entre 12,86 (P3) e 45,13 (P97) para as meninas e 16,13 (P3) e 53,66 (P97) para os meninos. Isso significa que na mesma idade há crianças vouzelenses com baixa proficiência motora (abaixo do P10) e outras que apresentam valores próximos ou superiores ao percentil 50 dos indivíduos com 10 anos de idade. Importa ressaltar que o aumento do desempenho coordenativo ao longo da idade tem magnitudes específicas em cada percentil. Nessa prova, meninas e meninos com valores de desempenho situados no P3 aumentam apenas 6,42 e 5,43 pontos, respectivamente, enquanto no $\mathrm{P} 97$ a progressão dos ganhos é de 31,87 pontos para as meninas e 18,06 pontos para os meninos. Um comportamento similar é verificado nos estudos desenvolvidos na Região Autônoma dos Açores $^{17}$ e no Peru ${ }^{18}$, embora a magnitude desses valores seja inferior à amostra vouzelense. Nas outras provas da bateria, a diferença dos percentis também é elevada e o aumento do desempenho coordenativo ao longo da idade é superior nos percentis mais elevados (P90 e P97), com exceção dos meninos na prova do ER, onde o aumento é constante e situa-se entre 10,31 e 11,24 pontos, considerando 
todos os percentis. Mediante tais evidências, atribuir significado e perceber o alcance da presença de forte variabilidade do desempenho coordenativo, em diferentes contextos socioeconômicos, educativos e culturais, torna-se uma tarefa ainda mais urgente dos profissionais da Educação Física no planejamento das suas estratégias educativas relativamente ao desenvolvimento da coordenação motora, com impacto nas aprendizagens cognitivas.

Em geral, as crianças vouzelenses apresentaram valores médios inferiores de desempenho coordenativo nas diferentes provas da bateria de testes KTK relativamente às crianças belgas e alemãs. Não obstante, nas provas em que o equilíbrio, o ritmo e a agilidade são mais requeridas, ER e TL, os valores médios das crianças vouzelenses situam-se próximos da amostra belga, enquanto os alemães apresentam valores superiores em todas as idades e em ambos os sexos. Considerando a defasagem temporal, i.e 35 anos entre o estudo alemão e os demais, essas diferenças poderiam ser atribuídas, eventualmente, ao efeito da tendência secular negativa no desempenho motor ocorrida em países europeus. São poucos os estudos sobre as mudanças seculares do desempenho motor de crianças ${ }^{31,32}$ e quase inexistentes quando o assunto é o desempenho coordenativo. Não obstante esta insuficiência e algum conflito entre resultados ${ }^{33}$, há evidência do declínio de componentes de aptidão física e neuromotora. ToMKINSON ${ }^{34}$, numa análise sistemática dos resultados reportados em 27 países de cinco regiōes geográficas, no período de 1958 a 2003, sugere uma tendência de declínio da performance (i.e., tendência secular negativa) em testes de velocidade ("sprint running and agility running tests"), entre outras capacidades, de crianças com idades compreendidas entre seis e 12 anos. No mesmo sentido, RUNHAAR et al. ${ }^{32}$ salientam uma redução da aptidão neuromotora de crianças holandesas, com idades entre os nove e os 12 anos, no período de 1980 e 2006. E ainda no domínio da aptidão física e competência motora, NisHIJIMA et al. ${ }^{35}$ reportam, também, uma tendência negativa, i.e., decréscimo contínuo dessas características em uma amostra japonesa $(\mathrm{n}=12.832)$, a partir de 1985 , tendo desenvolvido o estudo ao longo de 34 anos (1964-1997).

Nas tarefas motoras em que se requerer, além de capacidades coordenativas, a força muscular (explosiva) para uma boa performance, i.e, SM e SL, as crianças vouzelenses apresentam valores médios inferiores comparativamente às belgas e alemãs. Importa ressaltar a similitude do desempenho coordenativo nessas provas entre as crianças belgas e alemãs, a diferença temporal de 35 anos não impede que alguns valores médios da amostra belga se sobressaiam à alemã. Entre as crianças belgas e as vouzelenses, as diferenças tendem a aumentar ao longo da idade, variando entre 9,6 (meninos aos seis anos) e 32,56 pontos (meninos aos 10 anos) nos SM e entre 2,27 (meninos aos seis anos) e 20,03 pontos (meninos aos 10 anos) nos SL, em que as crianças belgas apresentam desempenho superior em todas as idades e em ambos os sexos. Relativamente à amostra alemã, as crianças vouzelenses também têm um desempenho inferior, à exceção dos meninos aos seis anos, e aumento das diferenças ao longo da idade; a amplitude dessas diferenças situa-se entre 0,64 (meninos aos seis anos) e 33,28 pontos (meninas aos 10 anos) nos $\mathrm{SM}$ e entre 0,32 (meninos aos sete anos) e 24,41 pontos (meninas aos 10 anos). Essas evidências podem estar relacionadas a aspectos das rotinas de vida das crianças bem como a fatores socioculturais intrínsecos de cada população [sobre esta matéria ver BoUCHARD et al. ${ }^{7}$; MaLINA et al. ${ }^{36}$, sendo difícil verificar uma tendência secular do comportamento do desempenho coordenativo nessas provas, em que a força muscular se mostra uma forte componente. Importa destacar, ainda, que os níveis de proficiência motora estão, de certo modo, condicionados pelas rotinas diárias das crianças, assim como pelos seus níveis e padrões de prática de atividade física ${ }^{1}$, marcados pela sua permanência na escola, carga horária das aulas de educação física, o modo como as diferentes facetas do currículo são priorizadas e aplicadas, assim como, pela motivação intrínseca de cada criança e pelas condições dos materiais e métodos disponibilizados específicos de cada país, cultura e espaçamento temporal.

No que concerne ao comportamento do P50 nas provas da bateria de testes KTK (ER, SM e SL), sugere-se certa similitude do desempenho coordenativo entre as três amostras contrastadas, i.e. Vouzela, Peru e Região Autônoma dos Açores, cuja amplitude das diferenças situa-se, sobretudo, abaixo dos 5 pontos. É provável que o modo como as crianças interagem com o seu ambiente e vivenciam suas experiências motoras lúdicas informais e formais possam ser similares, tanto no âmbito escolar, desportivo, quanto no contexto social em que estão inseridas. Ao analisar aspectos socioeducacionais do Concelho de Vouzela e da Região Autônoma dos Açores, não é possível reportar expressivas diferenças, haja vista o seguimento da mesma proposta curricular e prática educativa. Do mesmo modo, em Lima, no Peru, a Educação Física é estabelecida 
como uma importante área da educação, inserida no programa curricular ${ }^{37}$.

As pseudo-curvas de velocidade da mediana sugerem alguma especificidade em cada prova da bateria de testes KTK no que se refere às mudanças anuais de pontuação (as velocidades são negativas), i.e, podem estar associadas a diferentes janelas de oportunidades de aprendizagens motoras. Em todas as provas há declives negativos ao longo da idade, com distintas tendências mas sempre de natureza não-linear, sugerindo alguma diferenciação entre sexos, corroborando estudos prévios que reportam diferenças entre meninos e meninas ${ }^{12,14,22,38}$. Não é de nosso conhecimento qualquer estudo prévio que tenha reportado curvas ou pseudo-curvas de velocidade para o desempenho coordenativo não sendo possível analisar o seu comportamento. Contudo, ganha consistência a sugestão da redução da velocidade a que se verificam os incrementos anuais da coordenação motora entre os seis e os 10 anos de idade.

O presente estudo apresenta algumas limitações. Em primeiro lugar, a dimensão amostral e representatividade dos seis anos de idade, embora estudos prévios $^{19,29}$ tenham reportado amostras com efetivos semelhantes. Ainda assim, o reduzido erro-padrão nas estimativas das curvas $\mathrm{L}, \mathrm{M}$ e $\mathrm{S}$ aos seis anos de idade confere segurança ao cálculo dos percentis. Em segundo lugar, há que salientar o problema sempre sério de comparações entre as populaçôes quando não se possui informação adicional, validada transculturalmente, para interpretar diferenças entre desempenhos das crianças. Não obstante estes pontos, há aspectos fortes do presente estudo que merecem referência: 1) a utilização de um método robusto, sofisticado e muito atual relativamente à análise estatística, com a relevante representação gráfica e numérica dos perfis configuracionais do desempenho coordenativo das diferentes provas da bateria de testes KTK; 2) apresentação de pseudo-curvas de velocidade dos valores medianos para as quatro provas da bateria de testes KTK, sugerindo informação sobre diferentes janelas de oportunidades de desenvolvimento coordenativo, dos seis aos 10 anos de idade, contribuindo para o estabelecimento de planejamento e organização mais esclarecida e eficiente da prática educativa do Professor de Educação Física.

Em conclusão, os valores de referência construídos pelo presente estudo sugerem a presença de forte variabilidade interindividual do desempenho coordenativo das quatro provas da bateria de testes KTK. Daqui a necessidade em considerar as diferenças de desempenho entre crianças como um espaço privilegiado para ação pedagógica em contexto escolar, sendo que ao professor de Educação Física cabe a responsabilidade de apresentar propostas significativas de experiências motoras diversificadas e diferenciadas. Relativamente aos valores médios de estudos internacionais, nomeadamente alemães e belgas, o desempenho das crianças vouzelenses é inferior. Nas provas $\mathrm{ER} \mathrm{e} \mathrm{TL}$, onde se requer mais equilíbrio, ritmo e agilidade, sugere-se a eventualidade de uma tendência secular negativa no desempenho motor de crianças europeias, considerando a defasagem temporal entre estudo alemão e os demais. As comparaçôes entre os valores do P50 das amostras vouzelense, peruana e açoriana sugerem similitude nos resultados. Por fim, as pseudo-curvas de velocidade construídas mostram uma redução da velocidade dos incrementos anuais do desempenho coordenativo, entre os seis e os 10 anos de idade, sendo específica de cada prova da bateria de testes KTK e entre meninos e meninas.

\section{Abstract}

Motor coordination of children: construction of centiles charts with LMS method by Cole and Green

The purposes of this study were 1) to construct reference values to KTK test battery; 2) to compare Vouzela children's motor coordination with those from other Portuguese sites and international references; 3 ) to present pseudo-velocity curves for each KTK test. The sample comprises 811 children aged six to 11 years. Motor coordination was assessed with the four tests of the KTK Battery. Centile charts were constructed using the LMS method implemented in LMSchartmarker Pro software. Pervasive interindividual differences were noted in all coordination tests. Mean values of Vouzela children's motor coordination were lower than German and Belgian samples. 50th percentile was similar among Vouzela, Peruvian and Azorean samples. Pseudo-velocity curves suggested a marked specificity to each test and sex, as well as decreasing of the coordinative gains per year.

KEY WORDS: Motor coordination; Reference charts; KTK. 


\section{Referências}

1. Cairney J, Hay JA, Faught BE, Hawes R. Developmental coordination disorder and overweight and obesity in children aged 9-14 y. Int J Obes. 2005;29:369-72.

2. Hands B, Larkin D. Physical fitness differences in children with and without motor learning difficulties. Eur J Spec Needs Educ. 2006;21:447-56.

3. Cairney J, Hay JA, Faught BE, Flouris A, Klentrou P. Developmental coordination disorder and cardiorespiratory fitness in children. Pediatr Exerc Sci. 2007;19:20-8.

4. Cantell M, Crawford SG, Tish Doyle-Baker PK. Physical fitness and health indices in children, adolescents and adults with high or low motor competence. Hum Mov Sci. 2008;27:344-62.

5. Cairney J, Hay J, Veldhuizen S, Missiuna C, Mahlberg N, Faught BE. Trajectories of relative weight and waist circumference among children with and without developmental coordination disorder. CMAJ. 2010;182:1167-72.

6. Lee WA. Neuromotor synergies as a basis for coordinated intentional action. J Mot Behav. 1984;16:135-70.

7. Bouchard C, Malina RM, Pérusse L. Genetics of fitness and physical performance. Champaign: Human Kinetics; 1997.

8. Laskowski ER, Newcomer-Aney K, Smith J. Proprioception. Phys Med Rehabil Clin N Am. 2000;11:323-40.

9. Malina RM. Motor development during infancy and early childhood: overview and suggested directions for research. Int J Sport Health Sci. 2004;2:50-66.

10. Etchepare LS, Pereira EF, Zinn JL. Educação física nas séries iniciais do ensino fundamental. Rev Educ Fís/UEM. 2003;14:59-66.

11. Portugal. Ministério da Educação. Organização curricular e programas - ensino básico: 1 Ciclo. Algueirão-Mem Martins: Departamento da Educação Básica; 2004.

12. Kiphard EJ, Schilling F. Köperkoordinationtest für Kinder. Weinheim: Beltz Test GmbH; 1974.

13. Kiphard EJ, Schilling F. Körperkoordinationtest für Kinder. 2: Überarbeitete und ergänzte Auflage. Weinheim: Beltz Test GmbH; 2007.

14. Lopes VP, Maia J, Silva RG, Seabra A, Morais FP. Estudo do nível de desenvolvimento da coordenação motora da população escolar (6 aos 10 anos de idade) da Região Autónoma dos Açores. Rev Port Cien Desp. 2003;3:47-60.

15. Collet C, Folle A, Pelozin F, Botti M, Nascimento JV. Nível de coordenação motora de escolares da rede estadual da cidade de Florianópolis. Motriz. 2008;14:373-80.

16. Gorla JI, Duarte E, Montagner PC. Avaliação da coordenação motora de escolares da área urbana do município de Umuarama-PR, Brasil. R Bras Ci Mov. 2008;16:57-65.

17. Vidal SM, Bustamante A, Lopes VP, Seabra A, Silva RG, Maia JA. Construção de cartas centílicas da coordenação motora de crianças dos 6 aos 11 anos da Região Autónoma dos Açores, Portugal. Rev Port Cien Desp. 2008;9:24-35.

18. Valdívia AB, Lara R, Espinoza CB, et al. Prontitud coordinativa: perfiles multivariados en función de la edad, sexo y estatus socio-económico. Rev Port Cien Desp. 2008;8:34-46.

19. Largo RH, Fischer JE, Rousson V. Neuromotor development from kindergarten age to adolescence: developmental course and variability. Swiss Med Wkly. 2003;133:193-9.

20. Rosique J, Rebato E. Comparative study of statural growth in Spanish populations. Am J Hum Biol. 1995;7:553-64.

21. Zemel BS, Johnston FE. Application of the Preece-Baines Growth-Model to Cross-Sectional Data: problems of validity and interpretation. Am J Hum Biol. 1994;6:563-70.

22. Vandorpe B, Vandendriessche J, Lefevre J, et al. The KorperkoordinationsTest fur Kinder: reference values and suitability for 6-12-year-old children in Flanders. Scand J Med Sci Sports. 2011;21:378-88.

23. Cole TJ, Green PJ. Smoothing reference centile curves: the LMS method and penalized likelihood. Stat Med. 1992;11:1305-19.

24. Pan H, Cole TJ. lmsChartMaker, a program to construct growth references using the LMS method. Version 2.54. London: Medical Research Council; 2011.

25. Royston P, Wright EM. Goodness-of-fit statistics for age-specific reference intervals. Stat Med. 2000; 19:2943-62.

26. Pan H, Cole TJ. A comparison of goodness of fit tests for age-related reference ranges. Stat Med. 2004;23:1749-65.

27. Van Buuren S, Fredriks M. Worm plot: a simple diagnostic device for modelling growth reference curves. Stat Med. 2001;20:1259-77.

28. Guo SS, Roche AF, Chumlea WC, Johnson C, Kuczmarski RJ, Curtin R. Statistical effects of varying sample sizes on the precision of percentile estimates. Am J Hum Biol. 2000;12:64-74.

29. McCarthy HD, Cole TJ, Fry T, Jebb SA, Prentice AM. Body fat reference curves for children. Int J Obes. 2006;30:598-602. 
30. Roelants M, Hauspie R, Hoppenbrouwers K. References for growth and pubertal development from birth to 21 years in Flanders, Belgium. Ann Hum Biol. 2009;36:680-94.

31. Matton L, Duvigneaud N, Wijndaele K, et al. Secular trends in anthropometric characteristics, physical fitness, physical activity, and biological maturation in Flemish adolescents between 1969 and 2005. Am J Hum Biol. 2007;19:345-57.

32. Runhaar J, Collard DC, Singh AS, Kemper HC, Van Mechelen W, Chinapaw M. Motor fitness in Dutch youth: differences over a 26-year period (1980-2006). J Sci Med Sport. 2010;13:323-8.

33. Smpokos EA, Linardakis M, Papadaki A, Lionis C, Kafatos A. Secular trends in fitness, moderate-to-vigorous physical activity, and TV-viewing among first grade school children of Crete, Greece between 1992/93 and 2006/07. J Sci Med Sport. 2012;15:129-35.

34. Tomkinson GR. Global changes in anaerobic fitness test performance of children and adolescents (1958-2003). Scand J Med Sci Sports. 2007;17:497-507.

35. Nishijima T, Kokudo S, Ohsawa S. Changes over the years in physical and motor ability in Japanese youth in 1964-97. Int J Sport Health Sci. 2003;1:164-70.

36. Malina RM, Bouchard C, Bar-Or O. Growth, maturation, and physical activity. Champaign: Human Kinetics; 2004. 37. Valdívia AB. Análisis interactivo de la coordinación motora, actividad física y del índice de masa corporal en escolares peruanos: estudio transversal en niños de ambos sexos de los 6 a los 11 años de edad [Tese]. Porto(PT): Universidade do Porto, Faculdade de Desporto; 2007.

38. Valdívia AB, Cartagena LC, Sarria NE, et al. Coordinación Motora: Influencia de la edad, sexo, estatus socio-económico y niveles de adiposidad en niños peruanos. Rev Bras Cineantropom Desempenho Hum. 2008;10:25-34.

ANEXO I - Valores das curvas LMS e respectivos erros-padrão (ep) em função do sexo e da idade.

ER: equilíbrio à retaguarda;

SM: saltos monopedais;

SL: saltos laterais;

TL: transposição lateral.

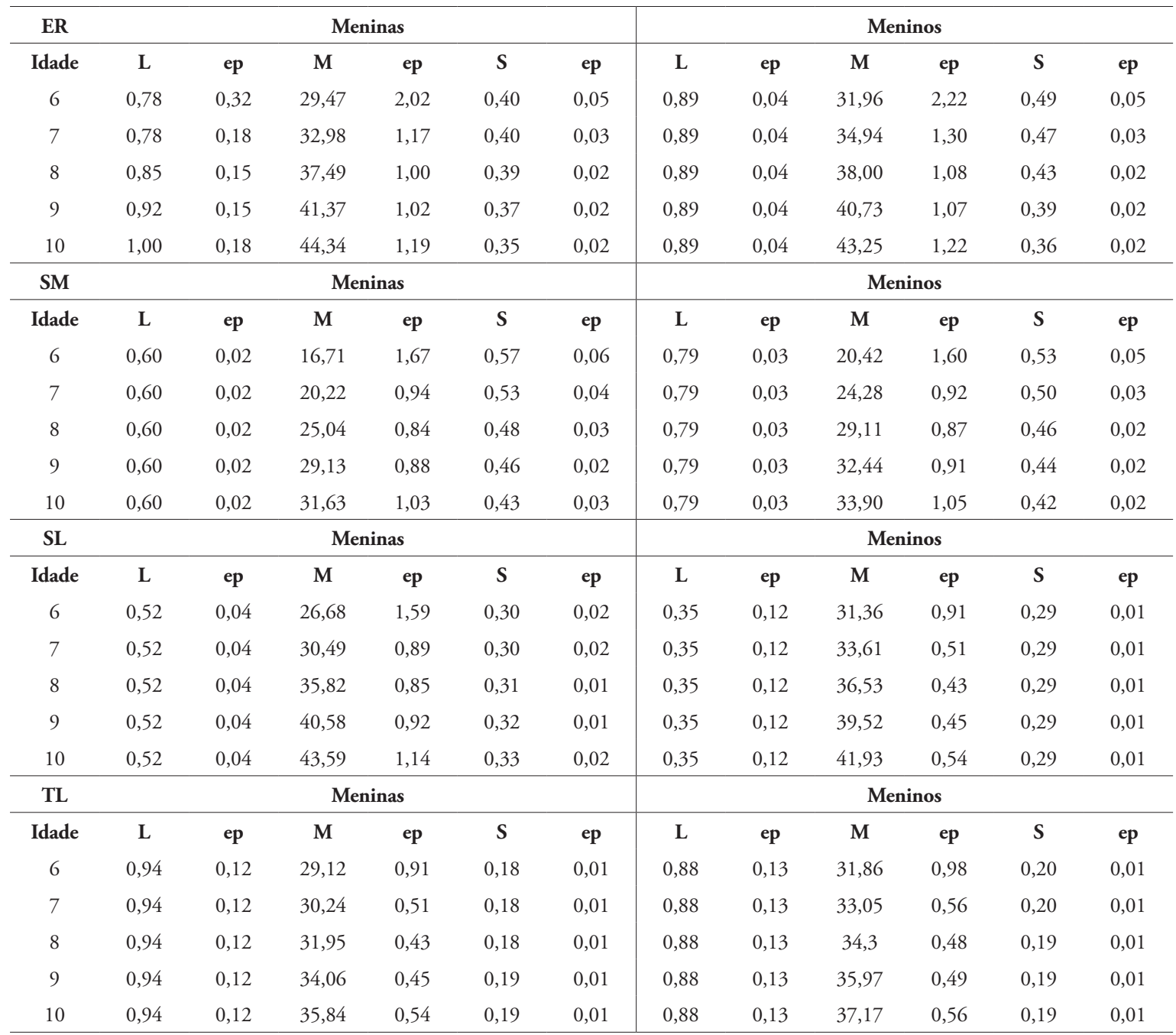

40 • Rev Bras Educ Fís Esporte, (São Paulo) 2013 Jan-Mar;27(1):25-41 


\section{Nota}

As autoras Raquel Nichele Chaves e Michele Caroline de Souza são bolsistas CAPES, Ministério da Educação, Brasil.

\section{Agradecimentos}

À Câmara Municipal de Vouzela, ao Agrupamento de escolas de Vouzela e a todos os profissionais da Educação Física e Desporto envolvidos no estudo; à Fundação para a Ciência e Tecnologia (FCT) de Portugal (financiamento ao projeto de referência PTDC/DES/67569/2006) e à Coordenação de Aperfeiçoamento de Pessoal de Ensino Superior - CAPES (bolsa de Doutorado no exterior/623110-1).

Um agradecimento especial a todas as crianças participantes do projeto "Vouzela Ativa".

Rachel Nichele de Laboratório de Cineantropometria e Estatística Aplicada Faculdade de Desporto

Universidade do Porto

R. Dr. Plácido Costa, 91 4200-450 - Porto - PORTUGAL Recebido para publicação: 17/05/2012 Revisão: 05/07/2012

Aceito: 10/07/2012 e-mail: raquelnichele@live.com.pt 